Rev. Elev. Méd. vét. Pays trop., 1974, 27 (1) : 75-80

\title{
Influence de la tuberculose sur le gain de poids de zébus à l'engrais
}

\author{
par J. M. BLANCOU et Y. CHENEAU (*)
}

\begin{abstract}
RESUME
Des différences importantes sont observées dans la moyenne des gains de poids entre 295 bovins sains et 442 bovins tuberculeux à divers stades. Ces différences sont d'autant plus grandes que la lésion tuberculeuse est plus grave. Elles peuvent atteindre 12 p. 100 dans le cas de l'embouche intensive (100 jours) et 28,7 p. 100 dans le cas de l'embouche à l'herbe (200 jours).
\end{abstract}

\section{INTRODUCTION}

La relation entre l'état tuberculeux de l'organisme et sa croissance pondérale est mal connue. Les études systématiques sur ce sujet sont très rares et ne concernent, à notre connaissance, que l'homme (4) ou les petits animaux de laboratoire (1).

A Madagascar, où la tuberculose bovine atteint selon BLANCOU et Collab., (3) environ le quart du cheptel, cette maladie cause de graves pertes économiques par mortalité et morbidité. L'affection entraîne souvent des troubles généraux graves dont la conséquence immédiate est un retard dans la croissance pondérale de l'animal. Cette question a déjà été abordée par SERRES et Collab, (5). C'est dans le but de chiffrer ce retard, avec autant de précision que possible, que nous avons comparé les gains de poids de 737 bovins à l'embouche en fonction des lésions tuberculeuses dont ils étaient porteurs à l'autopsie.

(*) I.E.M.V.T, Centre national de Recherches zootechniques et de Médecine vétérinaire, B.P. 862 , Tananarive, République Malgache.

\section{MATERIEL ET METHODES}

\section{Animaux d'expérience}

Il s'agit de bœufs Zébus d'embouche castrés, âgés de 4 à 8 ans répartis en :

1. Lots de bovins engraissés en parcs en 100 jours, avec des rations concentrées;

2. Lots de bœufs embouchés sur pâturage naturel, en 200 et 260 jours, ne recevant qu'une légère et occasionnelle complémentation sous forme de manioc.

Parmi ces 737 animaux, 442 (soit 60 p. 100) ont été trouvés porteurs de lésions tuberculeuses à l'autopsie.

Pour des raisons pratiques et économiques inhérentes à la situation zoo-sanitaire de Madagascar en matière de tuberculose, il n'est pas possible d'écarter de l'embouche les bovins réagissant à la tuberculine.

Nous avons dû éliminer de l'étude les résultats concernant 187 bovins embouchés à l'herbe en tant que porteurs de lésions de fasciolose.

\section{METHODE D'ETUDE}

Gains de poids. - On a considéré les dif- 
férences de poids entre la fin et le début de l'embouche pour chaque animal.

Autopsies. - Les lésions tuberculeuses recherchées avec soin lors de l'abattage ont été consignées sur des fiches de modèle unique.

\section{RESULTATS}

Les résultats des comparaisons de gains de poids sont exposés de la même façon, qu'il s'agisse de l'embouche intensive, ou d'embouche à l'herbe. Ils sont néanmoins étudiés séparément car l'incidence de la tuberculose varie selon la durée de la période d'étude (donc l'évolution de la maladie); de plus, les régimes alimentaires, donc les gains de poids, sont très différents selon que l'embouche est intensive ou non.

Les lésions ont été regroupées en :

- lésions viscérales, où l'atteinte des parenchymes pulmonaire et hépatique domine le tableau nécropsique;

- lésions ganglionnaires variées, affectant les divers groupes du système lymphatique de la carcasse ou des organes internes (ganglions céphaliques, intermusculaires, pulmonaires...).

Les résultats sont exposés aux tableaux I et II selon le type d'embouche. Dans ces tableaux, sont indiqués la moyenne des gains de poids des animaux malades et sains, l'erreur-standard et le résultat du test « $t$ » de Student à $t 0,05$.

L'étude statistique met en valeur des différences significatives entre les animaux sains et ceux atteints de tuberculose viscérale, quel que soit le mode d'embouche; par contre les lésions ganglionnaires n'influent pas significativement sur le gain de poids. La représentation graphique de l'influence de la tuberculose sur le gain de poids met en évidence les différences constatées plus haut (graphiques 1 et 2, p. 78).

\section{REMARQUES}

\section{Influence de la Iocalisation des lésions}

Disposant des relevés complets d'autopsies de chaque animal en expérience, nous avons pu distinguer, parmi les lésions viscérales, celles qui portaient atteinte aux organes de la cavité thoracique (tuberculose des poumons, de la plèvre...) et celles concernant la cavité abdominale.

Selon le mode d'embouche, ce sont soit les lésions viscérales thoraciques, soit les lésions viscérales abdominales qui influent significativement sur le gain de poids (tableau $\mathrm{n}^{\circ}$ III).

Ces différences peuvent s'expliquer par le fait que les bovins à l'embouche en parcs n'ayant pas à se déplacer ne font que peu appel à leur appareil respiratoire; par contre, ils doivent être en mesure d'assimiler de grandes quantités de nourriture : les lésions hépatiques, notamment, influent sur l'anabolisme. Il en va tout autrement pour les bovins au pâturage naturel: l'expérience s'est déroulée presque entièrement en saison sèche; les animaux, pour se nourrir et pour boire devaient parcourir plusieurs kilomètres dans la journée: aussi peut-on penser que sont intervenues alors en priorité les lésions pulmonaires et pleurales.

En ce qui concerne les lésions ganglionnaires, on n'observe pas de différence en les classant en lésions céphaliques, thoraciques, abdominales ou intermusculaires, et en étudiant chaque groupe séparément par rapport aux témoins.

\section{Evolution des gains de poids à plus long terme}

Une seconde pesée, réalisée à 260 jours sur des animaux à l'embouche au pâturage naturel, a donné les résultats indiqués au tableau $\mathrm{n}^{\circ} \mathrm{IV}$.

On constate que les lésions viscérales provoquent encore une perte de poids significative par rapport aux témoins, mais que toute différence entre les bovins atteints de tuberculose ganglionnaire et les animaux sains a disparu. Notons que les pluies ont débuté à la fin des 200 jours et que les bovins ont donc profité, dans les deux mois supplémentaires, d'une repousse importante de l'herbe.

\section{Influence de la fasciolose}

De nombreux bovins embouchés au pâturage naturel, dans une région où la fasciolose est très répandue, ont présenté à l'autopsie des lésions hépatiques graves. C'est ainsi que sur les 300 bovins de l'embouche en 200 jours, nous avons dû éliminer de l'étude précédente 103 résultats. Mais dans quelle mesure était-il néces- 
Retour au menu

TABLEAU N $N^{*}$ I

\begin{tabular}{|c|c|c|c|}
\hline \multirow{2}{*}{ Etat } & \multicolumn{2}{|c|}{ Embouche intensive (100 jours) } \\
\cline { 2 - 4 } & $\begin{array}{c}\text { Absence de } \\
\text { lësions }\end{array}$ & $\begin{array}{c}\text { Lếsions } \\
\text { viscérales }\end{array}$ & $\begin{array}{c}\text { Lésions } \\
\text { ganglionnaires }\end{array}$ \\
\hline Effectif & 71 & 57 & 61,10 \\
\hline $\begin{array}{c}\text { Moyenne des gains } \\
\text { de poids (kg) }\end{array}$ & 64,21 & 56,50 & 2,379 \\
\hline Erreur standard & 2,345 & 2,935 & $\begin{array}{c}0,9 \\
\text { Test "t" }(0,05)\end{array}$ \\
\hline
\end{tabular}

TABLEAU $N^{\circ}$ II

\begin{tabular}{|c|c|c|c|}
\hline \multirow{2}{*}{ Etát } & \multicolumn{2}{|c|}{ Embouche à l'herbe (200 jours) } \\
\cline { 2 - 4 } & $\begin{array}{c}\text { Absence de } \\
\text { lésions }\end{array}$ & $\begin{array}{c}\text { Lésions } \\
\text { viscérales }\end{array}$ & $\begin{array}{c}\text { Lésions } \\
\text { ganglionnaires }\end{array}$ \\
\hline Effectif & 90 & 39 & 68 \\
\hline $\begin{array}{c}\text { Moyenne des gaing } \\
\text { de poids (kg) }\end{array}$ & 33,90 & 24,15 & 29,20 \\
\hline Erreur standard & 2,168 & 4,323 & 2,330 \\
\hline Test "t" (0,05) & & $\begin{array}{c}2 \text { 2 24 } \\
\text { S1gnificatif }\end{array}$ & Non significatif \\
\hline
\end{tabular}

TABLEAU $N^{\circ}$ III

\begin{tabular}{|c|c|c|c|c|c|c|}
\hline \multirow[b]{2}{*}{ Types de lésions } & \multicolumn{3}{|c|}{ Embouche intensive ( 100 jours) } & \multicolumn{3}{|c|}{ Embouche à 1'herbe, (200 jour6) } \\
\hline & $\begin{array}{c}\text { Absence } \\
\text { de } \\
\text { lêsions }\end{array}$ & $\begin{array}{l}\text { Lésions } \\
\text { viscérales } \\
\text { thoraciques }\end{array}$ & $\begin{array}{l}\text { Lésions } \\
\text { viscêrales } \\
\text { abdoninales }\end{array}$ & $\begin{array}{c}\text { Absence } \\
\text { de } \\
\text { lésions }\end{array}$ & $\begin{array}{l}\text { Lésions } \\
\text { viscérales } \\
\text { thoraciques }\end{array}$ & $\begin{array}{l}\text { Lésions } \\
\text { viscérales } \\
\text { abdominales }\end{array}$ \\
\hline Effectif & 71 & 46 & 11 & 90 & 29 & 10 \\
\hline $\begin{array}{l}\text { Moyenne des gains } \\
\text { de poids (kg) }\end{array}$ & 64,21 & 58,23 & 49,27 & 33,90 & 23,17 & 27,00 \\
\hline Test "t" $(0,05)$ & & $\begin{array}{c}1,54 \\
\text { Non } \\
s i \text { gificatif }\end{array}$ & $\begin{array}{c}2,25 \\
\text { Significatif }\end{array}$ & & $\begin{array}{c}2,14 \\
\text { Significatif }\end{array}$ & $\begin{array}{c}1,04 \\
\text { Non } \\
\text { significatif }\end{array}$ \\
\hline
\end{tabular}

TABLEAU $N^{\circ}$ IV

\begin{tabular}{|l|c|c|c|}
\hline \multirow{2}{*}{ Etat } & \multicolumn{2}{|c|}{ Embouche à 1'herbe (260 jours) } \\
\cline { 2 - 4 } & $\begin{array}{c}\text { Absence de } \\
\text { lésions }\end{array}$ & $\begin{array}{c}\text { Lésions } \\
\text { viscérales }\end{array}$ & $\begin{array}{c}\text { Lésions } \\
\text { gang1ionnaires }\end{array}$ \\
\hline Effectif & 62 & 28 & 57 \\
\hline $\begin{array}{c}\text { Moyenne des gains } \\
\text { de poids (kg) }\end{array}$ & 38,45 & 26,17 & 39,75 \\
\hline Erreur standard & 3,097 & 4,676 & 2,805 \\
\hline Test "t" $(0,05)$ & & $\begin{array}{c}2,20 \\
\text { Significatif }\end{array}$ & Non significatif \\
\hline
\end{tabular}


EMBOUCHE A L'HERBE (200jours)
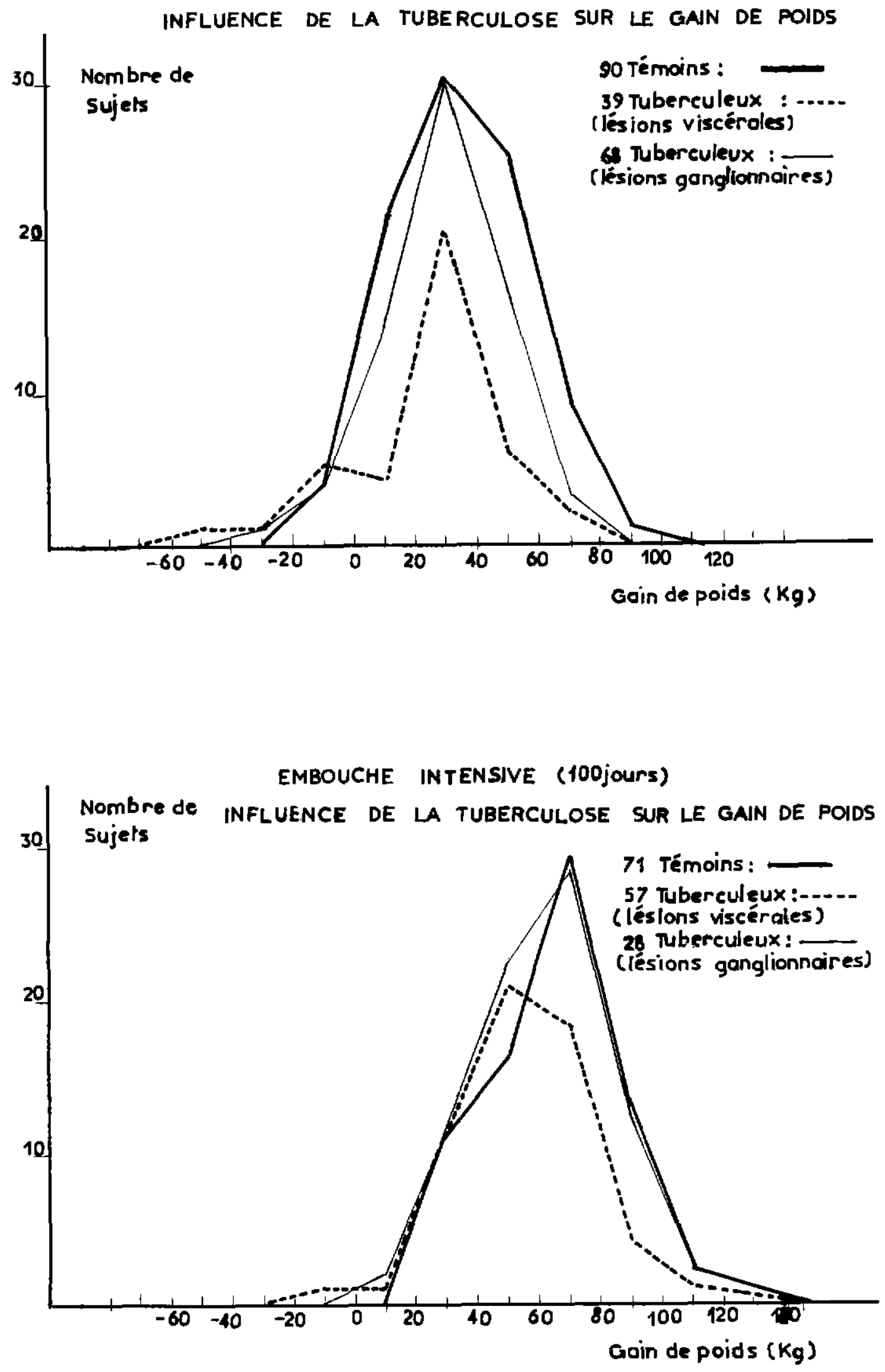
saire de procéder ainsi ? Les chiffres exposés dans le tableau $n^{\circ} \mathrm{V}$ ne concernant que des animaux indemnes de tuberculose, mais dont certains étaient atteints de distomatose, montrent une différence de gain de poids significative en faveur des sujets indemnes de tuberculose et de fasciolose.

TABLEAU N* V

\begin{tabular}{|l|c|c|}
\hline & \multicolumn{2}{|c|}{ Embouche en 200 jours } \\
\cline { 2 - 3 } & $\begin{array}{c}\text { Tëmoins } \\
\text { sains }\end{array}$ & $\begin{array}{c}\text { Sujets indemnes de } \\
\text { Tuberculose atteints } \\
\text { de Fasciolose }\end{array}$ \\
\hline Effectif & 90 & 42 \\
\hline $\begin{array}{c}\text { Moyenne des gains } \\
\text { de poids (kg) }\end{array}$ & 33,90 & 25,47 \\
\hline Erreur standard & 2,168 & 3,169 \\
\hline Test "t" & & $\begin{array}{c}2,19 \\
\text { Significatif }\end{array}$ \\
\hline
\end{tabular}

A la lecture du tableau $n^{\circ} \mathrm{V}$, il apparaît que la fasciolose influe de façon significative sur le gain de poids des bovins à l'engrais. Cette incidence aurait donc interféré avec celle de la tuberculose, aussi avons-nous écarté les résultats de tous les sujets atteints de fasciolose.

\section{DISCUSSION}

La présente étude n'a pas l'ambition de représenter l'ensemble des pertes dues à la tuberculose au cours des périodes indiquées car :

1. Un certain nombre de sujets $(3,1$ p. 100$)$ sont morts de tuberculose, ou ont dû être éliminés par abattage au cours de l'embouche et n'y figurent donc pas.

2. Il n'est pas tenu compte des pertes économiques par saisies lors de l'inspection sanitaire des viandes.

Les tableaux I et II permettent uniquement de chiffrer le manque à gagner qu'entraîne la présence de sujets tuberculeux lors d'une opération d'engraissement de bœufs.

En embouche intensive, le manque à gagner par rapport aux témoins s'élève à $7,71 \mathrm{~kg}$ par tête (soit 12 p. 100) pour les sujets atteints de lésions viscérales, et à $3,11 \mathrm{~kg}$ par tête (soit
4,8 p. 100 ) pour les bœufs atteints de tuberculose ganglionnaire.

Lors de l'embouche à l'herbe, la perte est de $9,75 \mathrm{~kg}$ par tête $(28,7$ p. 100$)$ du gain pour le groupe des sujets porteurs de lésions viscérales.

On peut ainsi chiffrer le risque économique créé par le facteur * tuberculose » dans les opérations d'embouche.

A Madagascar, comme dans plusieurs pays tropicaux, toute opération d'embouche faite sans tri préalable serait amenée à introduire un certain pourcentage de sujets tuberculeux (5). Pour éviter le manque à gagner consécutif, il existe deux solutions, de coût et de valeur inégales :

\section{Première solution}

Eliminer tous les sujets réagissant à la tuberculine, qui détectera 97 p. 100 des malades (2). Cela devrait éviter, théoriquement, presque tout le manque à gagner. Malheureusement, parmi les 3 p. 100 de malades non détectés par la tuberculination (anergiques) se trouve une majorité des sujets contagieux. L'on est alors ramené au problème précédent, surtout en embouche de longue durée, car la tuberculose est d'autant plus virulente qu'elle s'installe sur un sujet sain.

\section{Seconde solution}

$\mathrm{Ne}$ pas tuberculiner, mais viser uniquement à éliminer les sujets atteints de tuberculose avancée. L'examen clinique biochimique et sérologique (2) permet de détecter environ la moitié de ces malades. Les autres sont laissés dans le troupeau où l'effet de contagion est réduit par la barrière que représentent les sujets atteints de formes ganglionnaires, bénignes.

\section{CONCLUSIONS}

La tuberculose influe de façon très nette sur le gain de poids des bovins en embouche intensive, bien que ces différences ne soient pas statistiquement significatives lorsque les lésions sont cantonnées au système ganglionnaire.

L'influence est plus importante encore en embouche à l'herbe, où les perturbations physiologiques (en particulier au niveau de l'appareil respiratoire) sont plus durement ressenties. 
Du point de vue pratique, il paraît cependant difficile d'éviter ces pertes par la méthode de tuberculination en pays d'enzootie tuberculeuse. La méthode d'élimination sur examen clinique et sérologique paraît plus efficace.

\section{Remerciements}

Nous remercions vivement notre confrère Alexis Perdrix, Chef du Service Vétérinaire et Zootechnique de l'O.D.E.M.O., qui nous a apporté son précieux concours sur le terrain.

\section{SUMMARY}

Influence of tuberculosis on weight gains of fattening steers

Average live weight gains of 295 healthy and 442 steers at various stages of tuberculosis evolution, have shown noticeable differences. The greater the extension of the lesions is, the bigger the difference accounted for, and can reach up to 12 per cent for intensive fattening (100 days) and 28,7 per cent for pasture fattening (200 days).

\section{RESUMEN}

Influencia de la tuberculosis sobre el aumento de peso del cebú al engorde

Se observan diferencias importantes del término medio de los aumentos de peso entre 295 bovinos sahos y 442 bovinos a varios estados de tuberculosis.

Estas diferencias son tanto más grandes cuanto la lesión tuberculosis es más grave. Pueden llegar a 12 p. 100 en lo concerniente al engorde intensivo (100 días) y 28,7 p. 100 al engorde a pasto (200 días).

\section{BIBLIOGRAPHIE}

1. ANGLESIO (D.). Comportamento dell peso corporeo dalla cavia nell'infezione tubercolare sperimentale. B. batt. Immun., 1942, 29 : 139-152.

2. BLANCOU (J.M.). Comparaison de techniques pratiques de diagnostic de la tuberculose bovine. Rev. Elev. Méd. vét. Pays trop., 1972, 25 (1): 29-35.

3. BLANCOU (J. M.), ROHRBACH (C.), PERDRIX (A.), CHOQUEL (P.) et ROSNER (G.). La tuberculose bovine à Madagascar. Rev. Elev. Méd. vét. Pays trop., 1971, 24 (4): 505-517.
4. EDWARDS (L. B.), LIVESAY (V. T.), ACQUAVINA (F. A.), PALMER (C.). Height, weight, tuberculous infection, and tuberculous disease. Arch. environment Health, U.S.A., 1971, 22 (1): 106-112.

5. SERRES (H.), MEISSONNIER (E.), GODET (G.). Embouche de zébus malgaches. Essais complémentaires. Rev. Elev. Méd. vét. Pays trop., 1972, 25 (4) : $551-568$. 